\title{
A many-objective evolutionary algorithm based on integrated strategy for skin cancer detection
}

\author{
Yang Lan ${ }^{1}$, Lijie Xie ${ }^{1}$, Xingjuan Cai ${ }^{1 *}$, Lifang Wang ${ }^{*}$ \\ ${ }^{1}$ School of Computer Science and Technology, Taiyuan University of Science and Technology \\ Taiyuan, Shanxi 030024 - China. \\ [e-mail: lanyangvip1020@163.com, lijiexie11@163.com,xingjuancai@163.com,wlf1001@163.com] \\ * Corresponding author: Xingjuan Cai, Lifang Wang
}

Received September 25, 2021; revised December 6, 2021; accepted December 19, 2021; published January 31, 2022

\begin{abstract}
Nowadays, artificial intelligence promotes the rapid development of skin cancer detection technology, and the federated skin cancer detection model (FSDM) and dual generative adversarial network model (DGANM) solves the fragmentation and privacy of data to a certain extent. To overcome the problem that the many-objective evolutionary algorithm (MaOEA) cannot guarantee the convergence and diversity of the population when solving the above models, a many-objective evolutionary algorithm based on integrated strategy (MaOEA-IS) is proposed. First, the idea of federated learning is introduced into population mutation, the new parents are generated through sub-populations employs different mating selection operators. Then, the distance between each solution to the ideal point (SID) and the Achievement Scalarizing Function (ASF) value of each solution are considered comprehensively for environment selection, meanwhile, the elimination mechanism is used to carry out the select offspring operation. Eventually, the FSDM and DGANM are solved through MaOEA-IS. The experimental results show that the MaOEA-IS has better convergence and diversity, and it has superior performance in solving the FSDM and DGANM. The proposed MaOEA-IS provides more reasonable solutions scheme for many scholars of skin cancer detection and promotes the progress of intelligent medicine.
\end{abstract}

Keywords: integrated strategy, intelligent medicine, many-objective evolutionary algorithm, skin cancer. 


\section{Introduction}

The progress of artificial intelligence (AI) has promoted the process of intelligence in the medical industry $[1,2]$. However, the fragmentation and privacy of data have become the key factors that restricting the advance of intelligent medicine [3]. Nowadays, the emergence of new technologies such as federated learning (FL) and deep generation model has alleviated the above problems to some extent $[4,5]$. Particularly, for skin cancer detection (SCD), it not only ensures the effective detection of skin cancer, but also protects the information privacy of patients, and provides an effective paradigm for the accurate prevention of skin cancer [6].

Skin cancer is a disease caused by the spread of cancerous cells that is not controlled by the immune system. As one of the most common forms of cancer, it has seriously affected physical and mental health of people [7, 8]. For this reason, many scholars actively explore the symptom detection of early skin cancer. At present, with the fleetly progress of AI, the detection of skin cancer has changed from the previous human eye observation to the detection of machine learning model, which saves a mass of medical resources [9, 10]. Younis et al. [11] made skin cancer was classified through employed deep learning, which introduced transfer learning and adjusted the MobileNet convolution neural network that trained in advance. Setiawan et al. [12] explored the influence of skin cancer image reduction and color dimensionality reduction on skin cancer detection, and the above influencing factors were classified by K-means clustering. Meanwhile, Sabri et al. [13] proposed the modified integrate learning strategy to classify skin cancer, which through extracting the shape, color and texture features of the lesion area, different machine learning methods were employed to classify these features. However, the above studies only consider improving the detection accuracy of skin cancer, but other performance of skin cancer classification often were ignored. Start from the practical problems, Cai et al. [6] proposed a federated deep generation model, and the MaOEA was used to solve above model. By considering many factors of the deep generation model, the quality of the generated image was improved. Then, the problem of skin cancer detection has been transformed into a many-objective optimization problem (MaOP).

MaOPs exist in various fields, and its essence is that each objective cannot be optimal at the same time, and each objective has its own weight $[14,15]$. To distribute these weights reasonably, many-objective evolutionary algorithm (MaOEA) has become an ideal solution method [16, 17].

At present, MaOEA focus on studying the evolutionary mechanism of the population and the convergence and diversity of solutions are improved by increased the pressure of solution selection [18-20]. NSGA-III [21] adopted the strategy based on the reference point and a non-dominant sorting strategy to make the solution distribute evenly on the non-dominant layer, which avoided falling into the local optimization. GrEA [22] was based on the grid, which increased the selection pressure of the algorithm by boundary domination and boundary difference. In addition, the knee point was introduced into KnEA [23] to improve the convergence of the solution and reduced the complexity of calculation. VaEA [24] employed angular vector to guarantee the homogeneity of solution set in environment selection, and the solution with worse convergence was substituted through other solutions. However, above evolutionary algorithms only guarantee the performance of a certain aspect of the solution, they cannot take into account the diversity and convergence of the solution at the same time. For this reason, an integration strategy that could maintain the multifaceted performance of the solution was proposed. 
In recent decades, according to the different characteristics of the problem, integration methods angles have been proposed from different, such as multi-search strategy integration, parameter setting integration and decision variable integration. Wang et al. [25] proposed an effective integration framework to solve multi-objective optimization problems (MOPs), which combined different evolutionary operators with selection mechanisms, and the performance of the algorithm was maintained through cooperation and competition among populations. Wang et al. [26] adopted different integration ideas and integrated eight different bat algorithm (BA) search modes to solve the single-objective complex DV-Hop location problem. Fan et al. [27] proposed a multi-objective integration algorithm based on parameter and mutation strategy, which was suitable for different stages of evolution and solving different optimization problems. Peng et al. [28] designed a multi-objective optimization algorithm with multi-modal attributes under the framework of co-evolution.

However, most of the existing integrated algorithms are employed to solve single-objective or MOPs, and few integrated algorithms are used to solve the MaOPs. At the same time, the dual generative adversarial network model and SCD model based on federated for skin cancer research have been transformed into many-objective optimization problems. The extant integrated algorithms cannot solve the above models more effectively. Therefore, to better solve the above MaOPs, a many-objective evolutionary algorithm based on integrated strategy is proposed. The specific contributions of our work are as follows:

(1) The idea of federated learning was introduced, an integration strategy of mating pool based on federation was proposed. The offspring were randomly divided, and each part was mutated after employing different mating selection operators, which improved the diversity of the population.

(2) In environment selection, the distance between each solution to the ideal point and the Achievement Scalarizing Function value of each solution were considered to guarantee the convergence and diversity of the solution, and through the elimination mechanism to eliminate the non-ideal solution.

(3) The proposed algorithm solved the federated skin cancer detection model and dual generative adversarial network model, which shown superior performance in skin cancer detection, and further promoted the development of intelligent medicine.

The remainder of this article is summarized below. In Section II, background be introduced in detail, including many-objective evolutionary algorithm, FSDM and DGANM. The related methodology of MaOEA-IS is described in section III. The experimental results are displayed and interpretation in section IV. Finally, concludes our work in section V.

\section{Background}

\subsection{Many-objective evolutionary algorithm}

For most of the MaOPs, their objectives often conflict with each other, so it is difficult for ordinary methods to balance these objectives to obtain the optimal solution set [29, 30]. Many-objective evolutionary algorithm (MaOEA) provides a good paradigm for solving the above problems, which makes coordination and compromise between objective to make the overall objectives as optimal as possible [31, 32]. In recent years, many scholars have also made great progress in MaOEA, which also promoted the development of industrial applications. MaOEA is described as follows:

$$
\begin{aligned}
& F(x)=\left(f_{1}(x), \ldots, f_{m}(x)\right) \\
& \text { s.t. } \quad x \in \Omega
\end{aligned}
$$


where $x$ denotes decision variable, $f_{1}, \ldots, f_{m}$ represents the objective function, and $\Omega$ is the decision space.

MaOEA makes all objectives as optimal as possible in a given region at the same time, and the solution of MaOEA is usually a reasonably distributed solution, which is the optimal solution set scattered on the optimal pareto front. Therefore, in MaOEA, many objectives are optimized simultaneously, and they often conflict with each other, so when solving a MaOP, through a certain strategy to achieve a balance between each objective, to obtain a set of optimal solution set.

\subsection{A many-objective dual generative adversarial networks model}

The traditional skin cancer detection needs a lot of patient data to make the detection model could have a better detection performance [33]. However, in reality, the information of patients in each hospital is insufficient, which makes the detection of skin cancer face severe challenges.

To approach above problem, a many-objective dual generative adversarial network model (DGANM) was proposed. The Frechet inception distance (FID), the loss of dual generation adversarial network, sharpness of images (SOI) and image diversity (DOI), were optimized at the same time to enhance the quality of the generated images. The four objectives of DGANM are described as follows:

The calculation method of first objective SOI is denotes in formula (2). Here $q\left(v_{w} \mid l\right)$ represents the possibility that image $l$ appertain classes $w$, and $v$ denotes label vector of these input images.

$$
S O I=-\sum_{w=1} q\left(v_{w} \mid l\right) \log \left[q\left(v_{w} \mid l\right)\right]
$$

The second objective function is DOI, and it is described in formula (3).

$$
D O I=-\sum_{w=1}^{c} q\left(v_{w}\right) \log \left[q\left(v_{w}\right)\right]
$$

where the $q\left(v_{w}\right)$ denotes probability of category $w$, and $c$ is the amount of categories.

And the loss is obtained by training DualGAN network, the last objective function is FID, which is represented in formula (4).

$$
F I D=\left\|\beta_{r}-\beta_{y}\right\|_{2}^{2}+t e\left(\sum r+\sum y-2\left(\sum r \sum y\right)^{\frac{1}{2}}\right)
$$

here $\beta_{r}$ and $\sum r$ represent average value and variance value of the Gaussian distribution of these generated images, respectively, $\beta_{y}$ is the average value of Gaussian distribution of these input images, $\sum y$ denotes the variance value of Gaussian distribution of these input images, and te represents the trace of matrix.

\subsection{The federated skin cancer detection model based on many-objective optimization}

In recent years, information privacy of people has gradually become the focus of scholars [34]. Similarly, the information privacy of data in the field of skin cancer research has become a top priority. To better avoid the disclosure of patient data information, FL is combined with skin cancer, a many-objective federated skin cancer detection model (FSDM) is proposed. Meanwhile, to further improve the performance of FSDM, communication cost, 
the accuracy and loss of global model, and Area Under ROC Curve (AUC) were optimized simultaneously.

Starting from the practical problems and considering the problem of patient privacy that was not considered in the previous skin cancer research, the FSDM is constructed and transformed into a MaOP, and above model is solved through the optimization algorithm. FSDM not only protects the privacy of patient information, but also makes skin cancer detection effective. The four objectives of FSDM are shown as follows.

The first objective function and the second objective function are global accuracy and global loss, respectively. They are obtained through the continuous communication interacting between server and clients until global model converges. And the third objective is communication cost, which is described in formula (5).

$$
\text { communication cost }=\sum_{c=1}^{c} \frac{a_{c}}{c}
$$

Here, $c$ denotes the $c$-th client, $c$ represents the total number of clients and $a_{c}$ is the quantity of $c$-th client model weights.

The last objective function is AUC, which represents the area between Receiver Operating Characteristic (ROC) curve and x-axis, and ROC could be denoted by True Positive Rate (TPR) as the ordinate axis and False Positive Rate (FPR) as the abscissa axis. TPR represents the percentage of positive samples that are classified as positive, and TPR represents the proportion of negative samples that are classified as positive.

\section{Proposed Methodology}

\subsection{An integration strategy of mating pool based on federation}

In recent years, many mating selection operators with superior performance have been proposed. The mating selection operators of different evolutionary algorithms have different global and local search patterns, and they have different superiority when disposing optimization problems with some different peculiarity [35, 36]. However, the existing evolutionary algorithms have a single evolutionary method, which cannot well maintain the diversity of population evolution. Therefore, mating pool was constructed by integrated evolutionary operator strategy, and to improve the diversity of population evolution, a mating pool integration strategy based on federation was proposed.

The federated integration strategy was described as follows. First, after the population initialized, the population was randomly divided into three parts. Then, for each part of the population, perform mutation after different mating selection operators were employed. There were three forms of operators were adopted, including rerandom generation, tournament selection and grid-based selection. Eventually, these population generated by the three mating selection operators were merged into a single population, which after mutating will be merged with the parent, so that the better offspring were selected as the initial population of environmental selection. The process of above evolution operator will be summarized in Algorithm 1. 


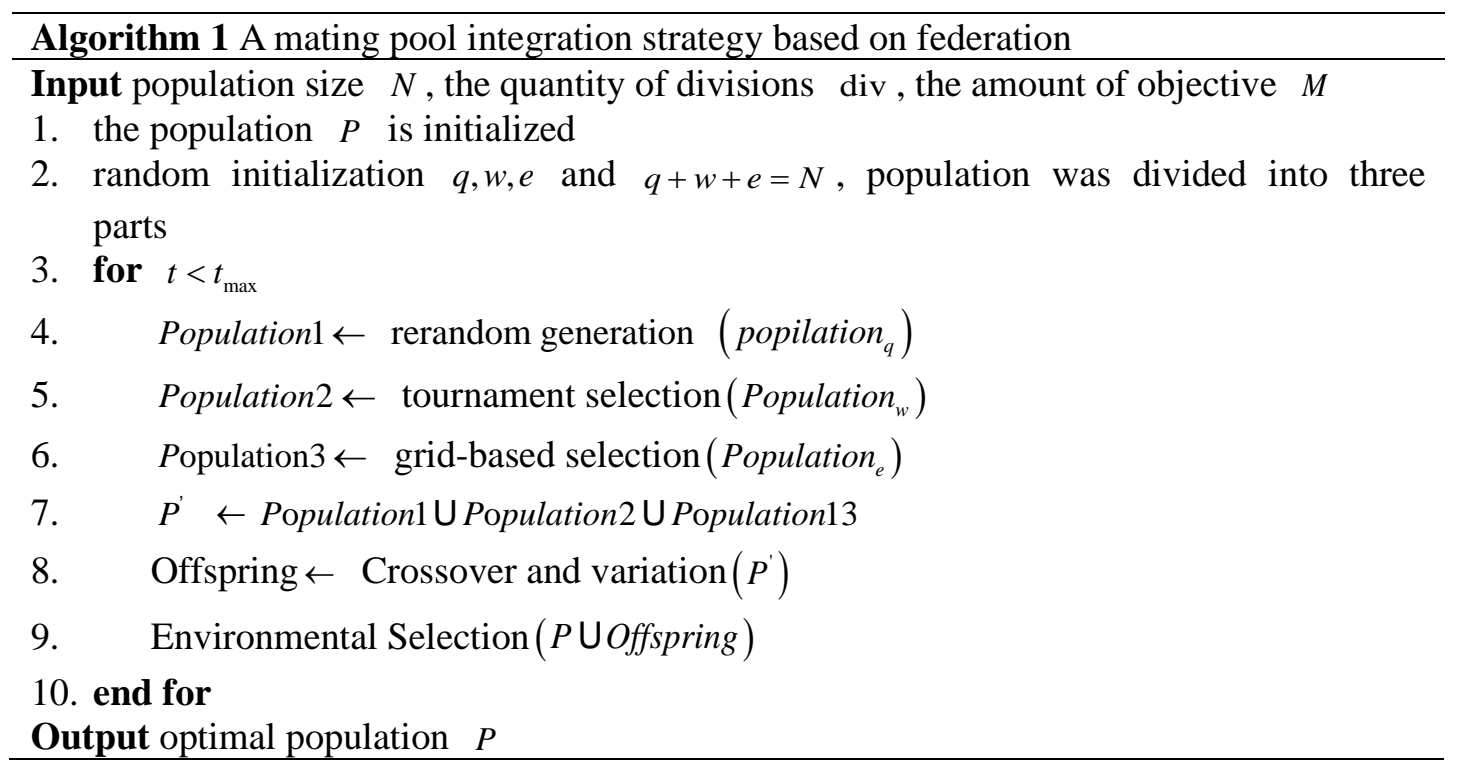

Where the $\leftarrow$ denotes latter assigns the result to the former after the relevant operation, and $U$ represents the union set of related elements.

\subsection{An integrated strategy of environment selection based on elimination mechanism}

At present, many novel and efficient algorithms have been proposed by scholars to solve MaOPs, such as many-objective evolutionary algorithms based on co-evolution and so on. These high-dimensional MaOEA are based on the convergence and diversity of the solution set, which to improve comprehensive performance of the solution set [37, 38].

However, different operators often lead to different algorithm performance, this makes different selection operators was chose when dealing with special optimization problems. Meanwhile, single operator insufficiently considers all the factors of the problem when solving MaOPs. The selection strategy pool was constructed through an integrated manner so that the selection operators were coordinated with each other. As a result, the overall evolutionary performance of the algorithm was improved, and significant distribution of the population were guaranteed at the same time. Therefore, an integrated strategy of environment selection based on elimination mechanism was proposed.

In our strategy, to guarantee better convergence and diversity of population, two methods were integrated to calculate the performance of the solution, including SID and ASF value of each solution. At the same time, considering the advantages and disadvantages of the two methods, the weight of SID was larger in the early evolution of the population and a large weight of ASF in the later population evolution. After obtaining the performance value of each solution, identify the two solutions with the minimum angle, which were selected to eliminate the solution of high value, while the solution of low value was retained in the next generation. The process of above environment selection will be described in detail in Algorithm 2. 


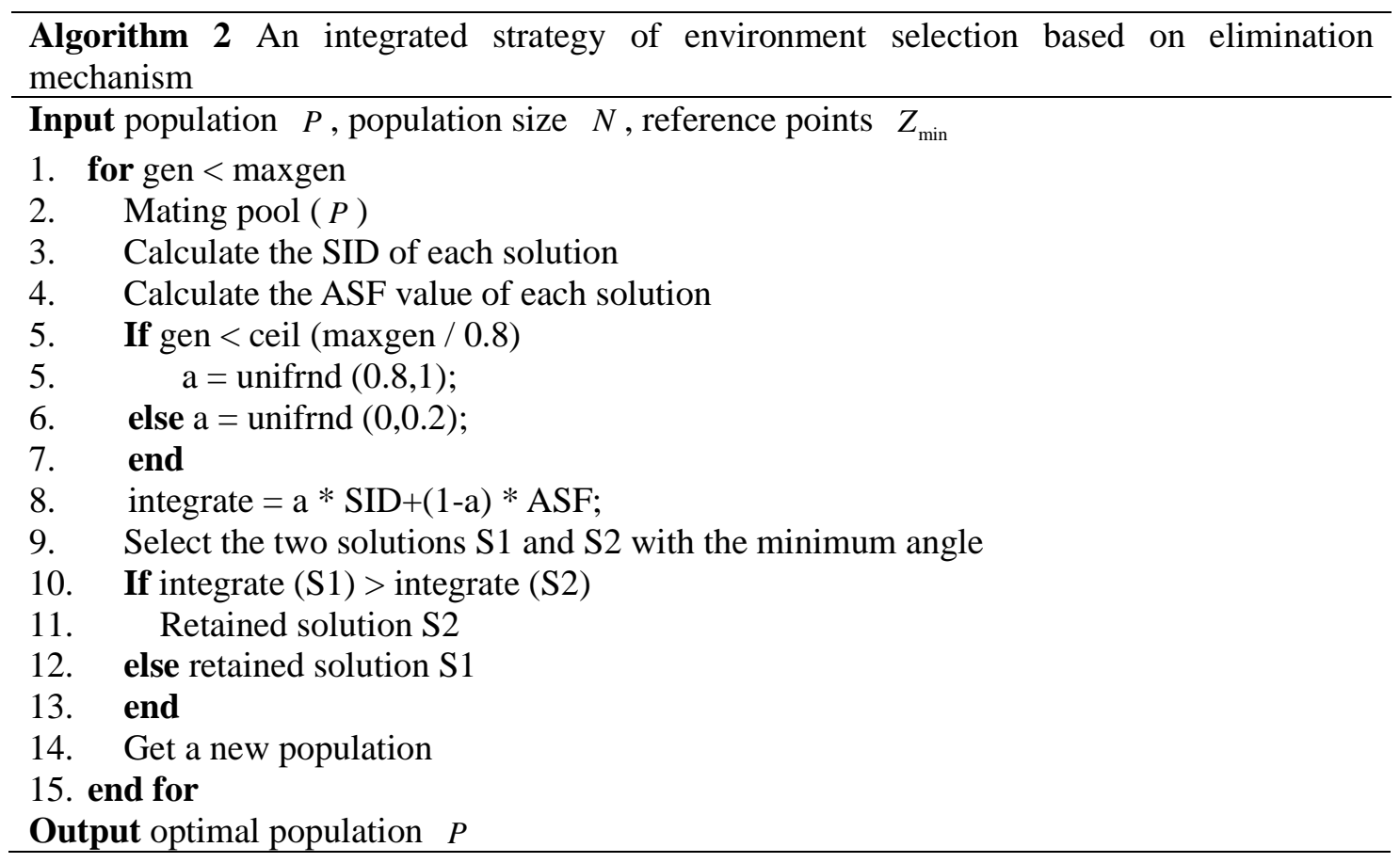

Where gen is the number of current iterations, maxgen denotes the maximum number of iterations and unifrnd is a function that generates random number in a specified interval.

\subsection{Many-objective evolutionary algorithm based on integration strategy}

In our study, to improve the comprehensive performance of traditional MaOEA, and better enough to solve proposed DGANM and FSDM. The many-objective evolutionary algorithm based on integration strategy (MaOEA-IS) was proposed. In our algorithm, the two key steps of evolutionary algorithm: mutation and selection, which were improved by the integrated strategy. Including an integration strategy of mating pool based on federation and an integrated strategy of environment selection based on elimination mechanism. The above methods enhance the global search ability of algorithm to some extent, ensure convergence and diversity of algorithm at the same time. The flow of MaOEA-IS is shown in Fig. 1.

As shown in the flow chart above, after initializing the population and relevant parameters of the algorithm, the mating selection strategy pool is adopted to generate $N$ offspring. Including rerandom generation, tournament selection and grid-based selection. After merging the parents and offspring, we select $N$ individuals as parents of the next generation through selection strategy pool. Including the SID and the ASF. Repeating the above steps until the condition is satisfied, then the evolution is over. 


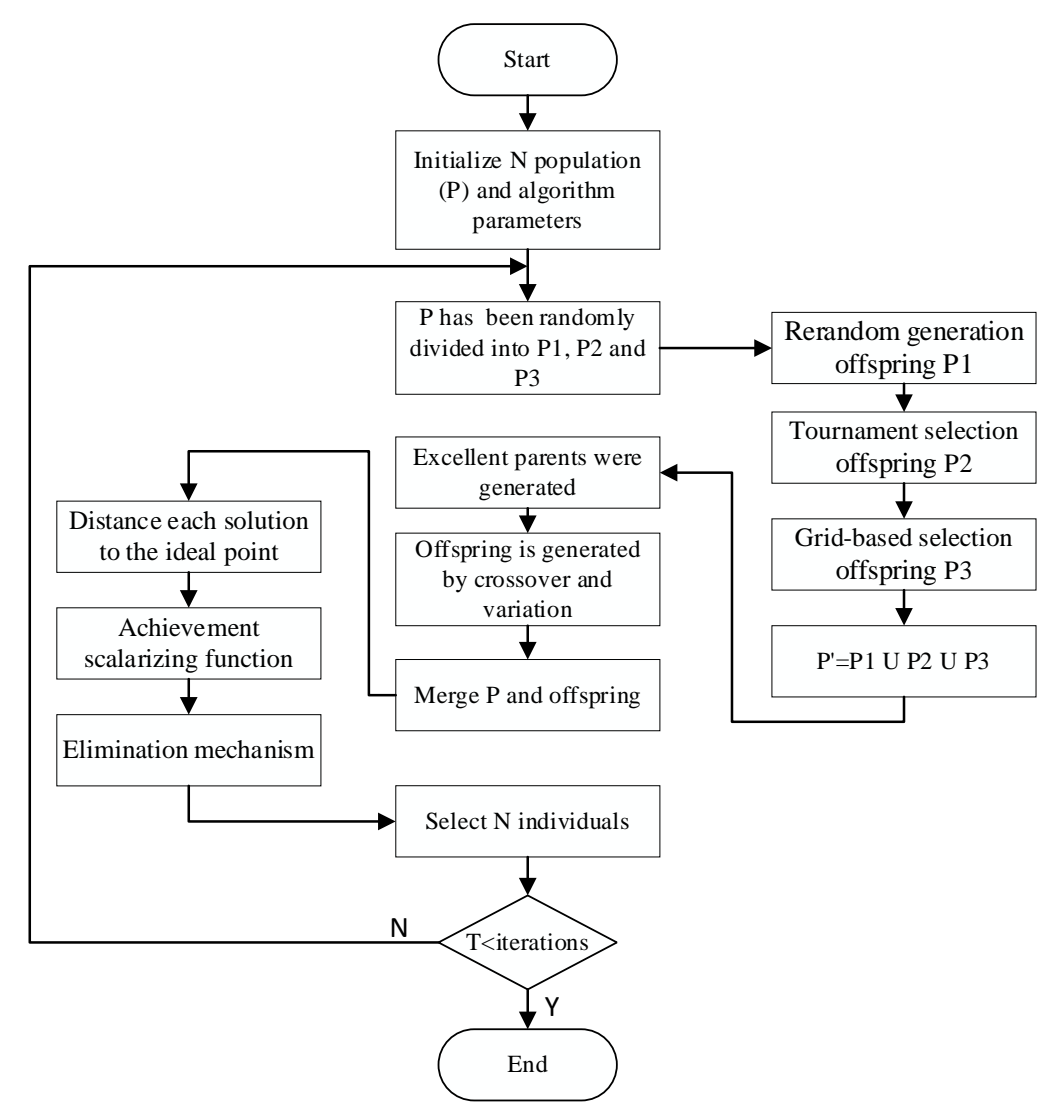

Fig. 1. The flow of the MaOEA-IS

\section{Experimental results}

\subsection{The performance of MaOEA-IS for Benchmark Problems}

\subsubsection{Experimental Settings}

In this section, the performance of the proposed MaOEA-IS is test through widely employed benchmark set DTLZ (DTLZ1-DTLZ7). The decision variable in DTLZ is set to $n=W+t-1$, here $W$ is the quantity of objectives that is set to $W \in(4,5,8,10,15)$. For different DTLZ, value of $t$ is different. In DTLZ2-DTLZ6, $k$ is 10, while in DTLZ1 and DTLZ7, $k$ is 5 and 20, respectively.

Meanwhile, to further test the performance of MaOEA-IS, five algorithms (including GrEA, NSGA-III, EFRRR [39], KnEA and VaEA) are employed to compare the performance in DTLZ. The parameter of six algorithms as shown follows: the cross probability $p c$ and the mutation rate $p m$ are 1 and $1 / D$, here $D$ denotes the amount of variables. And it in each objective of GrEA div is 15, the proportion of the population that becomes the knee point of KnEA rate is 0.5, the number of nearest weight vectors of EFRRR $v$ is 2. In addition, the initial population size of each algorithm is set to 20 and the population is iterated 30 times independently. 


\subsubsection{The Performance of MaOEA-IS in Benchmark Problems}

The six algorithms run 30 times independently on DTLZ, and the comparison results are shown in Table 1.

Table 1. The comparisons result of MaOEA-IS with five algorithms

\begin{tabular}{|c|c|c|c|c|c|c|c|}
\hline Problem & M & GrEA & NSGA-III & EFRRR & KnEA & VaEA & MaOEA-IS \\
\hline \multirow{5}{*}{ DTLZ1 } & 4 & $4.1093 \mathrm{e}-1(2.29 \mathrm{e}-1)+$ & $4.7162 \mathrm{e}-1(2.78 \mathrm{e}-1)+$ & $1.6510 \mathrm{e}+0(7.21 \mathrm{e}-1)-$ & $2.9073 e-1(1.74 e-1)+$ & $5.5007 e-1(2.57 e-1)+$ & $9.2370 \mathrm{e}-1(5.25 \mathrm{e}-1)$ \\
\hline & 5 & $1.1631 e+0(4.97 e-1)+$ & $1.4550 \mathrm{e}+0(4.64 \mathrm{e}-1)+$ & $6.2224 \mathrm{e}+0(2.32 \mathrm{e}+0)-$ & $8.2140 \mathrm{e}-1(3.92 \mathrm{e}-1)+$ & $1.4487 \mathrm{e}+0(4.54 \mathrm{e}-1)+$ & $2.1577 \mathrm{e}+0(9.34 \mathrm{e}-1)$ \\
\hline & 8 & $1.3567 e+0(6.42 e-1)=$ & $2.2887 e+0(1.05 e+0)-$ & $8.8993 e-1(3.36 e-1)=$ & $4.6540 \mathrm{e}+0(3.00 \mathrm{e}+0)-$ & $2.4969 e+0(1.33 e+0)-$ & $1.1539 \mathrm{e}+0(7.27 \mathrm{e}-1)$ \\
\hline & 10 & $3.6389 e+0(1.58 e+0)=$ & $4.1406 \mathrm{e}+0(1.62 \mathrm{e}+0)-$ & $1.8026 e+0(7.73 e-1)+$ & $2.5724 \mathrm{e}+0(1.11 \mathrm{e}+0)=$ & $3.8986 \mathrm{e}+0(1.32 \mathrm{e}+0)-$ & $2.7374 \mathrm{e}+0(8.51 \mathrm{e}-1)$ \\
\hline & 15 & $1.1914 \mathrm{e}+1(1.01 \mathrm{e}+1)-$ & $1.4714 \mathrm{e}+0(7.41 \mathrm{e}-1)-$ & $6.7370 \mathrm{e}-1(3.72 \mathrm{e}-1)=$ & $6.8472 \mathrm{e}+0(7.13 \mathrm{e}+0)-$ & $2.5099 \mathrm{e}+0(1.23 \mathrm{e}+0)-$ & $9.0142 \mathrm{e}-1(5.05 \mathrm{e}-1)$ \\
\hline \multirow{5}{*}{ DTLZ2 } & 4 & $1.3462 \mathrm{e}-1(2.22 \mathrm{e}-3)-$ & $1.2405 e-1$ (7.45e-4) - & $1.3047 \mathrm{e}-1$ (1.66e-3) - & 1.4066e-1 (4.43e-3) - & $1.2683 \mathrm{e}-1(1.21 \mathrm{e}-3)-$ & $1.1974 \mathrm{e}-1(1.30 \mathrm{e}-3)$ \\
\hline & 5 & 1.7694e-1 (2.38e-3) - & $1.8587 \mathrm{e}-1$ (3.01e-3) - & 2.0633e-1 (5.65e-3) - & $1.7752 \mathrm{e}-1(1.94 \mathrm{e}-3)-$ & $1.9369 \mathrm{e}-1$ (4.46e-3) - & $1.6500 \mathrm{e}-1(1.32 \mathrm{e}-3)$ \\
\hline & 8 & 4.0252e-1 (1.86e-2) - & 4.2758e-1 (1.03e-1) - & 3.8915e-1 (7.48e-3) - & $3.7815 e-1$ (1.00e-2) - & $4.5301 \mathrm{e}-1(2.39 \mathrm{e}-2)-$ & 3.5786e-1 (6.09e-3) \\
\hline & 10 & 4.1785e-1 (6.11e-3) - & 5.6489e-1 (6.44e-2) - & 4.7891e-1 (1.01e-2) - & $4.5337 \mathrm{e}-1$ (1.27e-2) - & 6.1513e-1 (3.61e-2) - & 4.1294e-1 (1.15e-2) \\
\hline & 15 & 6.0086e-1 (3.00e-2) - & 7.5341e-1 (5.14e-2) - & $5.7807 \mathrm{e}-1(1.56 \mathrm{e}-2)=$ & $6.1482 \mathrm{e}-1(1.11 \mathrm{e}-2)-$ & 7.4853e-1 (3.95e-2) - & $5.7311 \mathrm{e}-1(1.05 \mathrm{e}-2)$ \\
\hline \multirow{5}{*}{ DTLZ3 } & 4 & $1.2171 \mathrm{e}+1(5.37 \mathrm{e}+0)+$ & $2.1103 e+1(5.99 e+0)+$ & $3.8656 \mathrm{e}+1(1.11 \mathrm{e}+1)=$ & $9.6714 e+0(3.63 e+0)+$ & $1.6357 \mathrm{e}+1(5.53 \mathrm{e}+0)^{+}$ & $3.6565 e+1(1.03 e+1)$ \\
\hline & 5 & $4.3463 e+1(9.59 e+0)+$ & $5.3676 \mathrm{e}+1(7.93 \mathrm{e}+0)+$ & $9.5795 e+1(2.22 \mathrm{e}+1)-$ & $3.7775 e^{+1}(9.57 e+0)+$ & $5.3787 e+1(1.28 e+1)=$ & $6.0897 \mathrm{e}+1(1.04 \mathrm{e}+1)$ \\
\hline & 8 & $7.1332 \mathrm{e}+1(1.91 \mathrm{e}+1)-$ & $7.8137 \mathrm{e}+1(2.55 \mathrm{e}+1)-$ & $5.9539 \mathrm{e}+1(1.51 \mathrm{e}+1)-$ & $1.0329 \mathrm{e}+2(3.08 \mathrm{e}+1)-$ & $8.3784 \mathrm{e}+1(1.78 \mathrm{e}+1)-$ & $4.7424 e+1(8.80 e+0)$ \\
\hline & 10 & $2.0047 e+2(7.08 e+1)-$ & $1.3595 \mathrm{e}+2(3.21 \mathrm{e}+1)-$ & $9.1526 \mathrm{e}+1(1.86 \mathrm{e}+1)=$ & $1.3088 e+2(3.98 e+1)-$ & $1.3472 \mathrm{e}+2(2.61 \mathrm{e}+1)-$ & $8.6965 \mathrm{e}+1(1.90 \mathrm{e}+1)$ \\
\hline & 15 & $2.3956 \mathrm{e}+2(7.70 \mathrm{e}+1)-$ & $1.3244 \mathrm{e}+2(4.27 \mathrm{e}+1)-$ & $3.5403 e+1(1.10 e+1)=$ & $2.5246 \mathrm{e}+2(1.14 \mathrm{e}+2)-$ & $8.9463 \mathrm{e}+1(2.21 \mathrm{e}+1)-$ & $4.1310 \mathrm{e}+1(1.17 \mathrm{e}+1)$ \\
\hline \multirow{5}{*}{ DTLZ4 } & 4 & $1.8518 \mathrm{e}-1$ (1.18e-1) - & 1.7399e-1 (1.21e-1) - & $1.4635 \mathrm{e}-1$ (7.39e-2) - & $1.8960 \mathrm{e}-1$ (1.48e-1) - & $1.2736 \mathrm{e}-1(1.41 \mathrm{e}-3)-$ & $1.2227 \mathrm{e}-1(1.50 \mathrm{e}-3)$ \\
\hline & 5 & $1.8275 e-1$ (3.77e-3) - & $1.9352 \mathrm{e}-1$ (7.18e-3) - & 1.8745e-1 (3.03e-3) - & $1.9369 \mathrm{e}-1(2.88 \mathrm{e}-2)-$ & $1.9256 \mathrm{e}-1$ (4.94e-3) - & $1.7096 e-1(3.95 e-3)$ \\
\hline & 8 & 3.6276e-1 (4.28e-3) - & 4.2727e-1 (8.59e-2) - & 3.6757e-1 (2.18e-2) - & $4.5919 e-1(2.79 e-2)-$ & 4.7598e-1 (1.85e-2) - & $3.5527 \mathrm{e}-1(2.03 \mathrm{e}-3)$ \\
\hline & 10 & $4.2165 \mathrm{e}-1(3.41 \mathrm{e}-3)+$ & 5.8523e-1 (6.36e-2) - & 4.7642e-1 (1.38e-2) - & 5.4066e-1 (8.15e-3) - & 6.5706e-1 (2.36e-2) - & $4.3919 \mathrm{e}-1(1.86 \mathrm{e}-2)$ \\
\hline & 15 & 5.8874e-1 (3.68e-3) - & 7.8341e-1 (9.78e-2) - & 6.3170e-1 (2.05e-3) - & 6.4431e-1 (6.87e-3) - & 7.7673e-1 (2.49e-2) - & 5.7924e-1 (3.34e-3) \\
\hline \multirow{5}{*}{ DTLZ5 } & 4 & 8.7936e-2 (1.30e-2) - & 5.3990e-2 (1.40e-2) - & 1.2042e-1 (2.16e-2) - & $1.7105 \mathrm{e}-1$ (7.54e-2) - & 7.7322e-2 (1.62e-2) - & $4.5723 e-2(7.57 e-3)$ \\
\hline & 5 & $1.2375 e-1$ (3.48e-2) - & $2.1457 \mathrm{e}-1$ (7.57e-2) - & $2.5320 \mathrm{e}-1$ (3.55e-2) - & 2.8963e-1 (9.56e-2) - & 1.5191e-1 (1.76e-2) - & 7.5735e-2 (1.20e-2) \\
\hline & 8 & 2.7211e-1 (5.92e-2) - & $2.4583 e-1$ (9.50e-2) - & 3.2799e-1 (5.88e-2) - & 3.8526e-1 (1.06e-1) - & 3.4459e-1 (6.86e-2) - & $1.1498 \mathrm{e}-1(2.00 \mathrm{e}-2)$ \\
\hline & 10 & $3.2282 \mathrm{e}-1$ (4.82e-2) - & $2.1077 \mathrm{e}-1$ (3.92e-2) - & $3.1265 e-1$ (3.87e-2) - & $3.8729 \mathrm{e}-1$ (1.35e-1) - & 4.0709e-1 (7.54e-2) - & $1.4614 \mathrm{e}-1(2.13 \mathrm{e}-2)$ \\
\hline & 15 & 5.1327e-1 (8.04e-2) - & 3.0533e-1 (4.10e-2) - & 2.9550e-1 (5.24e-2) - & 5.5210e-1 (1.97e-1) - & 5.0977e-1 (1.20e-1) - & $1.2983 e-1(3.37 e-2)$ \\
\hline \multirow{5}{*}{ DTLZ6 } & 4 & $1.9630 \mathrm{e}-1(1.35 \mathrm{e}-1)=$ & 5.1756e-1 (5.20e-1) - & $2.5277 \mathrm{e}+0(6.99 \mathrm{e}-1)-$ & 8.1131e-1 (3.67e-1) - & 4.1163e-1 (2.89e-1) - & $2.5342 \mathrm{e}-1(2.77 \mathrm{e}-1)$ \\
\hline & 5 & 5.4179e-1 (3.23e-1) + & $3.8032 e+0(8.47 e-1)-$ & $5.9734 \mathrm{e}+0(4.58 \mathrm{e}-1)-$ & $1.4293 \mathrm{e}+0(3.83 \mathrm{e}-1)=$ & $3.8802 \mathrm{e}+0(4.51 \mathrm{e}-1)-$ & $1.5909 \mathrm{e}+0(6.02 \mathrm{e}-1)$ \\
\hline & 8 & $4.7131 \mathrm{e}+0(4.57 \mathrm{e}-1)-$ & $5.5536 \mathrm{e}+0(9.29 \mathrm{e}-1)-$ & $3.3104 \mathrm{e}+0(8.08 \mathrm{e}-1)-$ & $3.0440 \mathrm{e}+0(6.16 \mathrm{e}-1)-$ & $5.3361 \mathrm{e}+0(4.51 \mathrm{e}-1)-$ & $9.1068 e-1(3.67 e-1)$ \\
\hline & 10 & $3.7923 e+0(5.60 e-1)-$ & $6.7469 \mathrm{e}+0(4.50 \mathrm{e}-1)-$ & $5.7451 \mathrm{e}+0(6.50 \mathrm{e}-1)-$ & $3.2341 \mathrm{e}+0(5.87 \mathrm{e}-1)=$ & $6.3240 \mathrm{e}+0(4.70 \mathrm{e}-1)-$ & $3.0479 \mathrm{e}+0(6.46 \mathrm{e}-1)$ \\
\hline & 15 & $3.5020 \mathrm{e}+0(4.57 \mathrm{e}-1)-$ & $6.7141 \mathrm{e}+0(8.80 \mathrm{e}-1)-$ & $2.6331 \mathrm{e}+0(9.10 \mathrm{e}-1)-$ & $3.6158 \mathrm{e}+0(7.07 \mathrm{e}-1)-$ & $4.7003 e+0(3.79 e-1)-$ & $1.0994 e+0(4.10 e-1)$ \\
\hline \multirow{4}{*}{ DTLZ7 } & 4 & $1.6574 \mathrm{e}-1(9.78 \mathrm{e}-3)+$ & 3.4634e-1 (6.31e-2) - & 3.3961e-1 (4.38e-2) - & $2.2332 \mathrm{e}-1(1.36 \mathrm{e}-1)+$ & $2.7408 \mathrm{e}-1(6.29 \mathrm{e}-2)=$ & $3.2549 \mathrm{e}-1(1.22 \mathrm{e}-1)$ \\
\hline & 5 & 3.3194e-1 (3.11e-2) + & 7.8181e-1 (1.14e-1) - & 7.6526e-1 (8.39e-2) - & $3.9501 \mathrm{e}-1(6.03 \mathrm{e}-2)+$ & $6.0164 \mathrm{e}-1(5.71 \mathrm{e}-2)=$ & $6.9189 \mathrm{e}-1(2.37 \mathrm{e}-1)$ \\
\hline & 8 & $1.2006 \mathrm{e}+0(1.33 \mathrm{e}-1)-$ & $5.4850 \mathrm{e}+0(1.40 \mathrm{e}+0)-$ & $1.8592 \mathrm{e}+0(5.02 \mathrm{e}-1)-$ & $2.5511 e+0(1.05 e+0)-$ & $2.2489 \mathrm{e}+0(4.84 \mathrm{e}-1)-$ & $7.7667 \mathrm{e}-1(1.41 \mathrm{e}-1)$ \\
\hline & 10 & $6.9685 \mathrm{e}+0(9.57 \mathrm{e}-1)-$ & $1.3414 \mathrm{e}+1(1.26 \mathrm{e}+0)-$ & $8.6026 \mathrm{e}+0(1.12 \mathrm{e}+0)-$ & $9.0525 \mathrm{e}+0(2.29 \mathrm{e}+0)-$ & $1.0490 \mathrm{e}+1(1.43 \mathrm{e}+0)-$ & $1.9019 e+0(8.75 e-1)$ \\
\hline
\end{tabular}




\begin{tabular}{|c|c|c|c|c|c|c|}
\hline 15 & $1.8143 \mathrm{e}+1(1.83 \mathrm{e}+0)$ & $1.7632 \mathrm{e}+1(2.26 \mathrm{e}+0)-$ & $1.6046 \mathrm{e}+1(2.38 \mathrm{e}+0)-$ & $1.8099 \mathrm{e}+1(5.02 \mathrm{e}+0)-$ & $1.0239 \mathrm{e}+1(1.26 \mathrm{e}+0)-$ & $2.2907 \mathrm{e}+0(2.89 \mathrm{e}-1)$ \\
\hline$+1 /=$ & $8 / 24 / 3$ & $4 / 31 / 0$ & $1 / 28 / 6$ & $6 / 26 / 3$ & $3 / 29 / 3$ & \\
\hline
\end{tabular}

In Table 1, the labeled number is the best performance of the six algorithms in different dimensions under different test sets. “+”, “-” and " =” denote that the performance of this algorithm is better, worse and equal in a certain dimension, respectively, the total number of above symbols represent amount of comparison results than that of MaOEA-IS under the DTLZ test set. On the whole, the performance of MaOEA-IS is optimal when solving the problem of DTLZ, especially in solving the problem of DTLZ2, DTLZ4 and DTLZ5. According to the characteristics of the above test function, MaOEA-IS is better for solving degenerative, preference and concave problems. Although the performance superiority for proposed algorithm on DTLZ3, DTLZ6 and DTLZ7 is slightly worse than that of DTLZ2, DTLZ4 and DTLZ5, compared with other algorithms, the performance of MaOEA-IS is better on these test sets. It is not difficult to see that the performance of the MaOEA-IS is not very good on DTLZ1, because DTLZ1 focuses on the problem of multimodal and linear relationship, while our proposed model does not have the characteristics of these problem. Therefore, although the performance of the MaOEA-IS on DTLZ1 is slightly poor, it does not prove that the ability to solve the model is not excellent.

\subsection{The contrast experiments of different algorithms}

The performance of DGANM and FSDM are test through MaOEA-IS. And the ISIC-2018 dataset is employed to carry out above experiments. Next, the ISIC-2018 dataset and the parameter setting of DGANM and FSDM will be described in detail as follows. And the number of each category is shown in Table 2. Next, the parameter setting of DGANM and FSDM are introduced, respectively. For FSDM, the learning rate $\eta$ is set to 0.0001 , fraction of SET is set to 0.3 , local training times is set to 10 , batch size is set to 32 and the number of clients is set to 3. For DGANM, the number of neurons in three-layer convolution neural network of discriminator is 512, 256 and 1, respectively. The alpha of LeakyRelu is set to 0.2 , momentum of batchnormalization is set to 0.8 , and batch size of discriminator is set to 32 .

There are seven categories of ISIC-2018, including dermatofibroma (DF), melanocytic nevus (NV), basal cell carcinoma (BCC), actinic keratosis (AK), benign keratosis (BK), vascular disease (VASC) and melanoma (MEL), and its size is 10015.

Table 2. Original data distribution of the ISIC 2018

\begin{tabular}{cccc}
\hline Datasets & Class type & Amount & Total \\
\hline & AK & 327 & \\
BCC & 514 & 10015 \\
ISIC-2018 & BK & 1099 & \\
& DF & 115 & \\
& MEL & 1113 & \\
& NV & 6705 & 142 \\
\hline
\end{tabular}




\subsubsection{The performance of MaOEA-IS for DGANM}

The performance of different algorithms in solving DGANM will be compared, and to better analyze the results of each algorithm in population optimization, the boxplot will be drawn to observe the distribution of the optimal solution set after each algorithm optimizes the population. And the boxplot of five algorithms as shown in Fig. 2.

In Fig. 2, each line of the boxplot has the different meaning, with the minimum, the lower quartile, the median, the upper quartile, and the maximum from top to bottom, respectively. And the hollow circle also has its own meaning, which denotes the corresponding objective of individual in population is far greater than the general level, and it is the outlier of the population. As can been see from Fig. 2, the performance of the MaOEA-IS is superior in solving the DGANM. For each objective, except the loss, the median value of the MaOEA-IS is ideal, and it is the best among the five algorithms. And there are no outliers in solving DGANM for the proposed algorithm, which proves that the population distribution optimized by the MaOEA-IS is stable. Although the performance of MaOEA-IS is not ideal for the loss, its box size is the smallest, which proves that the distribution of the population on this objective is excellent. From this point of view, the performance of the proposed MaOEA-IS for loss is also acceptable. Therefore, above analysis proves that effect of MaOEA-IS in solving the model is excellent. Meanwhile, a pareto optimal solution set is provided by MaOEA-IS, and researchers could find the solution in the optimal solution set according to their own needs.
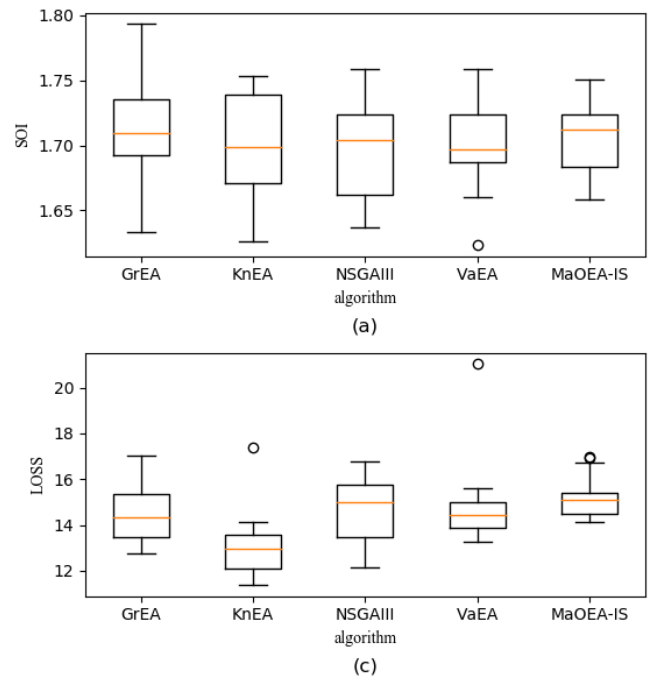

(c)

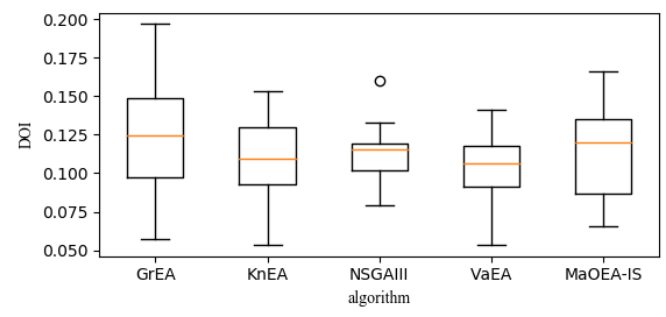

(b)

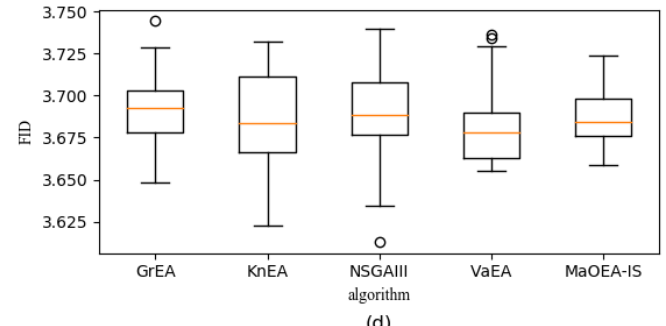

(d)

Fig. 2. The distribution of the solutions of four objectives on five algorithms.

To further display the performance of the MaOEA-IS on this model, individuals corresponding to different algorithms will generate images for follow-up experiments. Next, the comparison between original images and generated images will be shown in Fig. 3. For better comparison, the original images are converted into a grayscale image. 


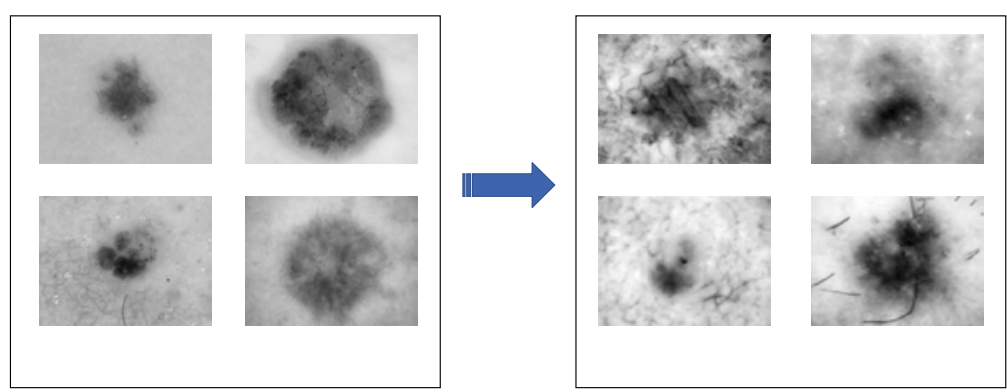

Fig. 3. The original images and the generated images

\subsubsection{The performance of MaOEA-IS for FSDM}

The performance of the five algorithms in solving FSDM will be compared, and results are shown in Table 3.

Table 3. Comparison consequence of different algorithms

\begin{tabular}{cccccc}
\hline Objective & GrEA & NSGA-III & VaEA & KnEA & MaOEA-IS \\
\hline ACC & 0.910160736 & 0.910264306 & 0.910314307 & 0.910471449 & 0.910550021 \\
Communication cost & 10620038.65 & 10305267.2 & 10348303.7 & 10202243.6 & 15110981.4 \\
AUC & 0.877639742 & 0.881585598 & 0.880633479 & 0.876167125 & 0.887299641 \\
LOSS & 0.255942235 & 0.254885349 & 0.254805297 & 0.257109947 & 0.252605089 \\
\hline
\end{tabular}

In Table 3, the corresponding objective value of each algorithm is the average value of all the solutions of the pareto solution set. Basically, the performance of the MaOEA-IS is excellent in three objectives, which proves that proposed algorithm has superior performance for solving the FSDM. In particular, for ACC, MaOEA-IS has the best result, which is $0.039 \%, 0.028 \%, 0.023 \%$ and $0.007 \%$ better than other algorithms, respectively. However, for Communication cost, the indicator value of MaOEA-IS is the worst. On the contrary, the result of KnEA is the best among these algorithms. The communication cost of MAOEA-IS is about 1.5 times than KnEA. And other algorithms are not much different from that of KnEA in this objective. For AUC, the performance of MaOEA-IS is $0.966 \%$ higher than GrEA, and the result of NSGA-III and VaEA are worse $0.571 \%$ and $0.667 \%$, respectively, the performance of KnEA is the worst, it is $1.113 \%$ worse than MaOEA-IS. It can be seen that the result of MaOEA-IS is the optimal on AUC. For LOSS, proposed algorithm makes the FSDM have the least loss. MaOEA-IS causes the loss of the model to be $0.334 \%$, $0.228 \%, 0.22 \%$ and $0.45 \%$ lower than other algorithms, respectively. In short, the performance of proposed algorithm is the best among five algorithms. MaOEA-IS achieves the best performance of other objective in this algorithm through expensive communication costs. The reason is that the proposed algorithm could get more weights when the convolution neural network is sparse, and it improves the complexity of neural network, which sacrifices training time in exchange for training accuracy, this situation is within the acceptable range. 


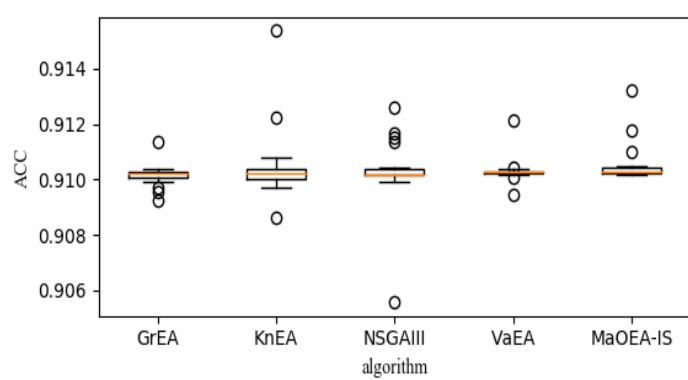

(a)

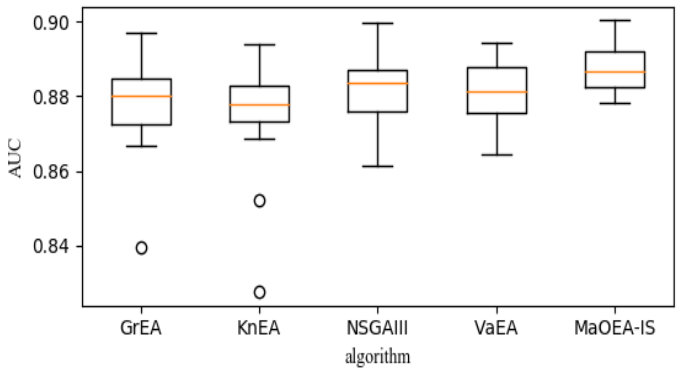

(c)

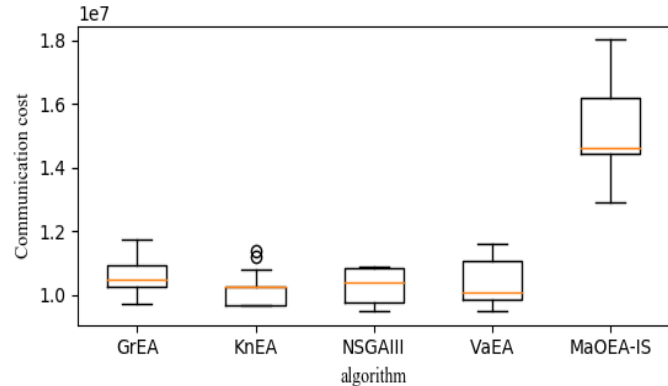

(b)

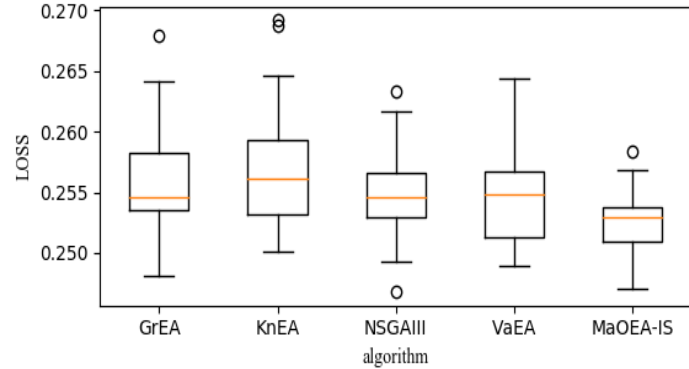

(d)

Fig. 4. The distribution of the solutions of four objectives on five algorithms.

To better demonstrate the performance of MaOEA-IS on optimal solution set, the boxplot of the optimal populations of the five algorithms are shown in Fig. 4. The five lines in the boxplot are explain in above experiment. And the hollow circle represents outlier. In Fig. 4, on the whole, except the poor distribution of the solution for the objective of communication cost, the population distribution of other objectives of MaOEA-IS is better than other algorithms. For ACC, although all algorithms have outliers, the boxplot of MaOEA-IS is relatively small. For AUC, the boxplot of MaOEA-IS has the largest AUC, and the median value is also the highest. Besides the size of its boxplot is also the smallest. For LOSS, the boxplot of MAOEA-IS is the minimum and has the least loss. In addition, the whole population of MaOEA-IS is distributed in places with lower values. However, for communication cost, the boxplot of MaOEA-IS is the worst, and the solution of MaOEA-IS is not well distributed on this objective. The reason for this situation has been given in the last experiment. In conclusion, the distribution of the optimized solution of the proposed algorithm is excellent, which also proves the superiority of MaOEA-IS in solving the FSDM.

\section{Conclusions}

In this study, to solve the DGANM and FSDM better, MaOEA-IS is proposed. Specifically, idea of federated learning is introduced into the mating pool, and different mating selection operators are employed for the offspring population that randomly divided, and after perform mutation then a new parent is obtained. For environmental selection, ASF and SID are used to guarantee convergence and diversity of population, in addition, elimination strategy is adopted to select a more appropriate solution as the parent of the next iteration. From the experimental results, the proposed MaOEA-IS achieves better performance on the DTLZ test set, DGANM and FSDM, and the above models could better be solved by MaOEA-IS while ensuring the diversity and convergence of the population. This study further promotes the research of skin cancer detection, and provides more reliable 
and effective solutions scheme for scholars, which promotes the rapid development of intelligent medicine.

\section{Acknowledgement}

This work is supported by the National Natural Science Foundation of China under Grant No.61806138; Key R\&D program of Shanxi Province (International Cooperation) under Grant No. 201903D421048; Key R\&D program of Shanxi Province (High Technology) under Grant No. 201903D121119; Key R\&D program (international science and technology cooperation project) of Shanxi Province, China under Grant No.201903D421003, Graduate Innovation Program of Shanxi Province under Grant No. 2021Y697.

\section{References}

[1] C. Guo, S. Su, K. R. Choo, and X. Tang, "A Fast Nearest Neighbor Search Scheme Over Outsourced Encrypted Medical Images," IEEE Transactions on Industrial Informatics, vol. 17, no. 1, pp. 514-523, Jan. 2021. Article (CrossRef Link).

[2] B.Wilson, J. P. M. Dhas, R. M. Sreedharan, and R. P. Krish, "Ensemble learning-based classification on local patches from magnetic resonance images to detect iron depositions in the brain" International Journal of Bio-Inspired Computation, vol. 17, no. 4, pp. 260-266, 2021. Article (CrossRef Link).

[3] Z. Cui, F. Xue, S. Zhang, X. Cai, Y. Cao, W. Zhang, and J. Chen, "A Hybrid BlockChain-Based Identity Authentication Scheme for Multi-WSN," IEEE Transactions on Services Computing, vol. 13, no. 2, pp. 241-251, 1 March-April 2020. Article (CrossRef Link).

[4] F. Sattler, S. Wiedemann, K. -R. Müller, and W. Samek, "Robust and Communication-Efficient Federated Learning From Non-i.i.d. Data," IEEE Transactions on Neural Networks and Learning Systems, vol. 31, no. 9, pp. 3400-3413, Sept. 2020. Article (CrossRef Link).

[5] K. Bin, S. Luo, X. Zhang, J. Lin, and X. Tong, "Compressive Data Gathering With Generative Adversarial Networks for Wireless Geophone Networks," IEEE Geoscience and Remote Sensing Letters, vol. 18, no. 3, pp. 558-562, March 2021. Article (CrossRef Link).

[6] X. Cai, Y. Lan, Z. Zhang, J. Wen, Z. Cui, and W. S. Zhang, "A Many-objective Optimization based Federal Deep Generation Model for Enhancing Data Processing Capability in IOT," IEEE Transactions on Industrial Informatics, 2021. Article (CrossRef Link).

[7] H. Y. Kim, H. Jung, H. M. Kim, and H. J. Jeong, "Surfactin exerts an anti-cancer effect through inducing allergic reactions in melanoma skin cancer," International Immunopharmacology, vol. 99, no. 107934, Oct 2021. Article (CrossRef Link).

[8] J. Hoehn A. Hekler, E. Krieghoff-Henning, JN. Kather, JS. Utikal, F. Meier, FF. Gellrich, A. Hauschild, L. French, J. Schlager, K. Ghoreschi, T. Wilhelm, H. Kutzner, M. Heppt, S. Haferkamp, W. Sondermann, D. Schadendorf, B. Schilling, R. Maron, M. Schmitt, T. Jutzi, S. Froehling, D. Lipka, and T. Brinker, "Integrating Patient Data Into Skin Cancer Classification Using Convolutional Neural Networks: Systematic Review," Journal of Medical Internet Research, Review, vol. 23, no. 7, pp. 14, Jul 2021. Article (CrossRef Link).

[9] Y. Jusman, I. M. Firdiantika, D. A. Dharmawan, and K. Purwanto, "Performance of Multi Layer Perceptron and Deep Neural Networks in Skin Cancer Classification," in Proc. of 2021 IEEE 3rd Global Conference on Life Sciences and Technologies (LifeTech), Nara, Japan, pp. 534-538, 9-11 March 2021. Article (CrossRef Link).

[10] M. G. Chen, W. J. Chen, W. Chen, L. Z. Cai, and G. Chai, "Skin Cancer Classification with Deep Convolutional Neural Networks," Journal of Medical Imaging and Health Informatics, vol. 10, no. 7, pp. 1707-1713, Jul 2020. Article (CrossRef Link). 
[11] H. Younis, M. H. Bhatti, and M. Azeem, "Classification of Skin Cancer Dermoscopy Images using Transfer Learning," in Proc. of 2019 15th International Conference on Emerging Technologies (ICET), Peshawar, Pakistan, pp. 1-4, 2-3 Dec. 2019. Article (CrossRef Link).

[12] A. W. Setiawan, A. Faisal, and N. Resfita, "Effect of Image Downsizing and Color Reduction on Skin Cancer Pre-screening," in Proc. of 2020 International Seminar on Intelligent Technology and Its Applications (ISITIA), Surabaya, Indonesia, pp. 148-151, 22-23 July 2020. Article (CrossRef Link).

[13] M. A. Sabri, Y. Filali, H. El Khoukhi, and A. Aarab, "Skin Cancer Diagnosis Using an Improved Ensemble Machine Learning model," in Proc. of 2020 International Conference on Intelligent Systems and Computer Vision (ISCV), Fez, Morocco, pp. 1-5, 9-11 June 2020. Article (CrossRef Link).

[14] X. Cai S. Geng, J. Zhang, D. Wu, Z. Cui, W. Zhang, and J. Chen, "A Sharding Scheme-Based Many-Objective Optimization Algorithm for Enhancing Security in Blockchain-Enabled Industrial Internet of Things," IEEE Transactions on Industrial Informatics, vol. 17, no. 11, pp. 7650-7658, Nov. 2021. Article (CrossRef Link).

[15] W. Deng, J. Xu, Y. Song, and H. Zhao, "An effective improved co-evolution ant colony optimisation algorithm with multi-strategies and its application" International Journal of Bio-Inspired Computation, vol. 16, no. 3, pp. 158-170, 2020. Article (CrossRef Link).

[16] Z. Cui, Y. Zhao, Y. Cao, X. Cai, W. Zhang, and J. Chen, "Malicious Code Detection under 5G HetNets Based on a Multi-Objective RBM Model," IEEE Network, vol. 35, no. 2, pp. 82-87, March/April 2021. Article (CrossRef Link).

[17] J. H. Li, G. Y. Chen, M. Li, and H. Chen, "An enhanced-indicator based many-objective evolutionary algorithm with adaptive reference point," Swarm and Evolutionary Computation, vol. 55, no. 100669, Jun 2020. Article (CrossRef Link).

[18] Z. J. Xiong, J. M. Yang, Z. Y. Hu, Z. W. Zhao, and X. J. Wang, "Evolutionary many-objective optimization algorithm based on angle and clustering," Applied Intelligence, vol. 51, no. 4, pp. 2045-2062, Apr 2021. Article (CrossRef Link).

[19] H. T. Zhao and C. S. Zhang, "An online-learning-based evolutionary many-objective algorithm," Information Sciences, vol. 509, pp. 1-21, Jan 2020. Article (CrossRef Link).

[20] S. R. Konda, L. K. Panwar, B. K. Panigrahi, R. Kumar, and V. Gupta, "Binary fireworks algorithm application for optimal schedule of electric vehicle reserve in traditional and restructured electricity markets," International Journal of Bio-Inspired Computation, vol. 18, no. 1, pp. 38-48, 2021. Article (CrossRef Link).

[21] K. Deb and H. Jain, "An Evolutionary Many-Objective Optimization Algorithm Using Reference-Point-Based Nondominated Sorting Approach, Part I: Solving Problems With Box Constraints," IEEE Transactions on Evolutionary Computation, vol. 18, no. 4, pp. 577-601, Aug. 2014. Article (CrossRef Link).

[22] S. X. Yang, M. Q. Li, X. H. Liu, and J. H. Zheng, "A Grid-Based Evolutionary Algorithm for Many-Objective Optimization," IEEE Transactions on Evolutionary Computation, Article vol. 17, no. 5, pp. 721-736, Oct 2013. Article (CrossRef Link).

[23] X. Y. Zhang, Y. Tian, and Y. C. Jin, "A Knee Point-Driven Evolutionary Algorithm for Many-Objective Optimization," IEEE Transactions on Evolutionary Computation, Article vol. 19, no. 6, pp. 761-776, Dec 2015. Article (CrossRef Link).

[24] Y. Xiang, Y. Zhou, M. Li, and Z. Chen, "A Vector Angle-Based Evolutionary Algorithm for Unconstrained Many-Objective Optimization," IEEE Transactions on Evolutionary Computation, vol. 21, no. 1, pp. 131-152, Feb 2017. Article (CrossRef Link).

[25] W. Wang, S. Yang, Q. Lin, Q. Zhang, K. Wong, C. Coello, and J. Chen, "An Effective Ensemble Framework for Multiobjective Optimization," IEEE Transactions on Evolutionary Computation, vol. 23, no. 4, pp. 645-659, Aug. 2019. Article (CrossRef Link).

[26] Y. Wang, P. Wang, J. Zhang, X. Cai, W. Li, and Y. Ma, "A novel DV-Hop method based on coupling algorithm used for wireless sensor network localization," International Journal of Wireless and Mobile Computing, vol. 16, no. 2, pp. 128-137, 2019. Article (CrossRef Link). 
[27] Q. Fan and X. Yan, "Differential evolution algorithm with self-adaptive strategy and control parameters for P-xylene oxidation process optimization," Soft computing, vol. 19, no. 5, pp. 1363-1391, May. 2015. Article (CrossRef Link).

[28] X. Peng, Y. Jin, and H. Wang, "Multimodal Optimization Enhanced Cooperative Coevolution for Large-Scale Optimization," IEEE Transactions on Cybernetics, vol. 49, no. 9, pp. 3507-3520, Sept. 2019. Article (CrossRef Link).

[29] Z. Zhang, Y. Cao, Z. Cui, W. Zhang, and J. Chen, "A Many-Objective Optimization Based Intelligent Intrusion Detection Algorithm for Enhancing Security of Vehicular Networks in 6G," IEEE Transactions on Vehicular Technology, vol. 70, no. 6, pp. 5234-5243, June 2021. Article (CrossRef Link).

[30] Y. Hu and X. Yan, "Neural network-assisted expensive optimisation algorithm for pollution source rapid positioning of drinking water," International Journal of Bio-Inspired Computation, vol. 17, no. 4, pp. 227-235, 2021. Article (CrossRef Link).

[31] Z. Zhang, M. Zhao, H. Wang, Z. Cui, and W. Zahng, " An efficient interval many-objective evolutionary algorithm for cloud task scheduling problem u nder uncertainty," Information Sciences, vol. 583, pp. 56-72, Jan, 2022. Article (CrossRef Link).

[32] X. Cai, S. Geng, D. Wu, J. Cai, and J. Chen, "A Multicloud-Model-Based Many-Objective Intelligent Algorithm for Efficient Task Scheduling in Internet of Things," IEEE Internet of Things Journal, vol. 8, no. 12, pp. 9645-9653, 2021. Article (CrossRef Link).

[33] Y. Xie, J. Zhang, Y. Xia, and C. Shen, "A Mutual Bootstrapping Model for Automated Skin Lesion Segmentation and Classification," IEEE Transactions on Medical Imaging, vol. 39, no. 7, pp. 2482-2493, July 2020. Article (CrossRef Link).

[34] C. Xu, A. S. Ding, and S. S. Liao, "A privacy-preserving recommendation method based on multi-objective optimisation for mobile users," International Journal of Bio-Inspired Computation, vol. 16, no. 1, pp. 23-32, 2020. Article (CrossRef Link).

[35] H. Chen, Y. Tian, W. Pedrycz, G. Wu, R. Wang, and L. Wang, "Hyperplane Assisted Evolutionary Algorithm for Many-Objective Optimization Problems," IEEE Transactions on Cybernetics, vol. 50, no. 7, pp. 3367-3380, July 2020. Article (CrossRef Link).

[36] J. Yuan, H. -L. Liu, F. Gu, Q. Zhang, and Z. He, "Investigating the Properties of Indicators and an Evolutionary Many-Objective Algorithm Using Promising Regions," IEEE Transactions on Evolutionary Computation, vol. 25, no. 1, pp. 75-86, Feb. 2021. Article (CrossRef Link).

[37] W. Na, S. Yuchao, C. Xiaohong, L. Xia, and L. Dui, "A $\in$-indicator-based shuffled frog leaping algorithm for many-objective optimization problems," Journal of Systems Engineering and Electronics, vol. 31, no. 1, pp. 142-155, Feb. 2020. Article (CrossRef Link).

[38] M. Xu, M. Zhang, X. Cai, and G. Zhang, "Adaptive neighbourhood size adjustment in MOEA/D-DRA," International Journal of Bio-Inspired Computation, vol. 17, no. 1, pp. 14-23, 2021. Article (CrossRef Link).

[39] Y. Yuan, H. Xu, B. Wang, B. Zhang, and X. Yao, "Balancing Convergence and Diversity in Decomposition-Based Many-Objective Optimizers," IEEE Transactions on Evolutionary Computation, vol. 20, no. 2, pp. 180-198, April 2016. Article (CrossRef Link). 


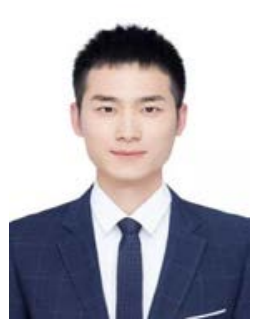

Yang Lan is currently working toward M.S. degree at computer science and technology, Taiyuan University of Science and Technology, Taiyuan, China. His research interests include computational intelligence, combinatorial optimization and deep learning.

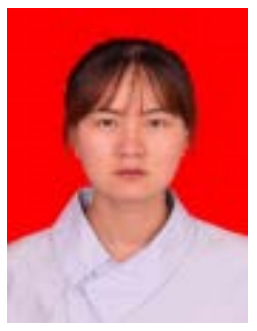

Lijie Xie is currently working toward M.S. degree at computer science and technology, Taiyuan University of Science and Technology, Taiyuan, China. Her research interests include computational intelligence, knowledge graph and explicable recommendation.

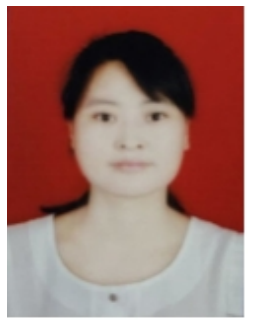

Xingjuan Cai received the Ph.D. degree in control theory and engineering from Tongji University, Shanghai, China, in 2017. She is a Professor with the School of Computer Science and Technology, Taiyuan University of Science and Technology, Taiyuan, China. Her interesting includes cloud computing, bio-inspired computation and applications.

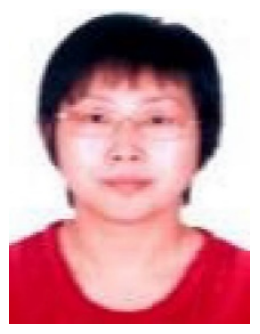

Lifang Wang received the Ph.D. degree in control theory and engineering from Lanzhou university of technology, Lanzhou China, in 2011. She is a Professor of Computer Science and Technology at Taiyuan University of Science and Technology, Taiyuan, China. She is engaged in research in intelligent computing. 